MODELING, IDENTIFICATION AND CONTROL, 1986, VOL. 7, NO. 1, 45-56

doi:10.4173/mic.1986.1.3

\title{
Adaptive control of alumina reduction cells with point feeders
}

\author{
PETER BORG†, TOM MOEN $\ddagger$ and JOHANNES AALBU $\ddagger$
}

Keywords: Adaptive control, alumina reduction, parameter estimation.

To obtain optimum operation of an alumina reduction cell, it is important to keep the alumina concentration in the cell within a certain range. This will reduce anode effect frequency and stabilize the cell operation. However, it is not possible to measure this concentration directly. In the system presented here, the characteristic nonlinear relation between the alumina concentration and the cell resistance is modelled by an adaptive linear model where the estimated parameters give information about the concentration. This information is then used to control the alumina supply to the cell. In addition, the system controls the energy input to the cell by keeping the average resistance close to a reference value.

The paper describes the methods used and some case studies done off-line, and summarizes the overall results from nearly three years' testing of the system on-line.

\section{Introduction}

The development of adaptive control of alumina to reduction cells has been carried out through a cooperation between A/S Årdal og Sunndal Verk (ÅSV) and Institute for energy technology (IFE), Norway. The practical experiments have been carried out on four test cells in line 1 in Årdal. These test cells were converted from side break-and-feed to point feeding in 1981.

The adaptive controller is a part of $\AA$ SV's point feeder technology, and is today in operation on three different types of cells in ÅV. In addition to the test cells where this control system was developed, there are $200 \mathrm{kA}$ and $220 \mathrm{kA}$ side-by-side cells operated on the same control strategy.

ÅSV and IFE have been working with estimation and control of alumina reduction cells for several years, and a multivariable control system based on a relatively complex state-space representation of the cell, was developed in the late seventies, Gran (1980). This system was run on one test cell for some time, but mainly due to the fact that the process was not fully observable through the measurements, the model had a tendency to drift off after a while. This work is now suspended, while the much simpler system described in this paper has been developed for the cells with point feeders. This feeder technique causes much less disturbance to the cell than the older side break-and-feed and centre-fed cells. This is the reason why it was assumed that the simpler control system would be adequate.

Received 13 February 1985. Revised 3 October 1985.

†Institute for energy technology, Kjeller, Norway.

$\ddagger$ A/S Årdal og Sunndal Verk, Årdalstangen, Norway. 


\section{The control problem}

The production of aluminium takes place in electrolytic cells where alumina is dissolved in a bath of molten cryolite. The alumina is reduced to aluminium at temperatures of $950-970^{\circ} \mathrm{C}$. For a brief description of the process, sec Gran (1980).

The current and the voltage of the cell are measured on-line and used to calculate the resistance. These are in fact the only on-line measurements. Other important states of the process, like the chemical composition and the temperature of the bath, are (presently) impossible to measure on-line. This is because both adequate on-line measurement techniques do not exist and because the cryolite will dissolve all metal-made sensors after some time, even if the sensor is encapsulated in ceramics.

Control of an alumina reduction cell is difficult due to this lack of measurements. The control system proposed here, includes an estimator part which extracts information about the concentration of alumina in the bath from the measured resistance. This information is then used to control the supply of alumina to the cell. In this context we may regard the estimator as an advanced measurement system, rather than an adaptive system in the usual sense.

In addition to controlling the alumina concentration, the controller should keep the resistance of the cell close to a reference value. This is necessary to stabilize the energy input to the cell.

The limits of the alumina concentration may be relatively broad without influencing the process in a negative way. When the concentration is too low, the socalled anode effect is introduced. This is characterized by a rapid and considerable increase in resistance due to the formation of gas bubbles under the anode. This phenomenon has been utilized in earlier control strategies to 'measure' the alumina concentration about twice a day, but this causes an extra energy consumption and also a major disturbance to the cell and should therefore be avoided as much as possible.

A too high concentration of alumina is also unwanted, because saturation is reached at about 7.5 to $8 \%$, and the alumina will deposit as sludge on the bottom of the cell. A favourable concentration band is therefore 2 to $5 \%$.

The variation in alumina concentration will cause variations in the resistance of the cell. These variations are intentional and should not cause frequent anode movements by the controller. However, the resistance may differ from the reference value for other reasons, for example because the interpolar distance has changed. To keep the average energy input to the cell within certain limits, it is necessary to adjust the anode position from time to time.

\section{Process modelling and estimation algorithm}

The theory of parameter estimation and adaptive control is well described in the literature, e.g. Isermann (1981), Ljung and Söderström (1983). We will here just give a brief summary relevant to our application.

The adaptive control algorithm consists of two parts; the parameter estimation and the controller calculation, as illustrated in Fig. 1. The separation of these two tasks usually follows the so-called certainty equivalence principle, which means that the calculation of the controller assumes that the estimated process parameters are the real ones. The controller calculation is therefore similar to conventional control with constant and known process parameters, and various controllers could be used dependent on the actual implementation. 


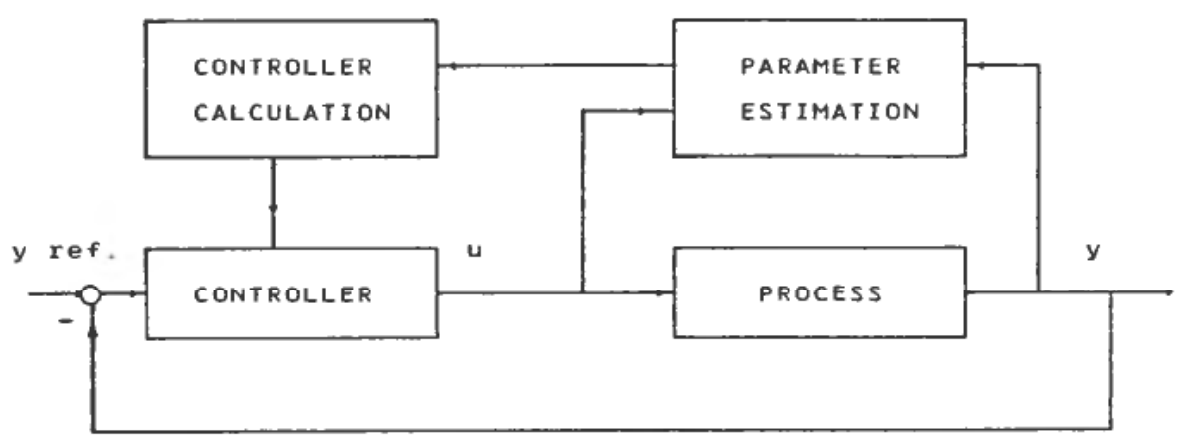

Figure 1. Structure of adaptive control systems.

Various parameter estimation techniques are reported in the literature. In this work we have considered the ordinary and extended least squares methods (RLS and RELS) and the recursive maximum likelihood method (RML).

To achieve reliable estimates, the process has to be persistently excited, which in loose terms means that the input signal to the process must excite all modes of the process, i.e. it must be sufficiently rich. This is often in conflict with the control purpose where one usually is interested in minimizing the deviations in output from a (constant) reference.

The estimation is based on an ARMAX (autoregressive-moving-averageexegenous-control) representation of the process, which for a single-input singleoutput system has the general form:

$$
A\left(z^{-1}\right) y(k)=z^{-j} B\left(z^{-1}\right) u(k)+D\left(z^{-1}\right) v(k)
$$

where

$$
\begin{array}{ll} 
& A\left(z^{-1}\right)=1+a_{1} z^{-1}+a_{2} z^{-2}+\cdots a_{n} z^{-n} \\
& B\left(z^{-1}\right)=b_{1} z^{-1}+b_{2} z^{-2}+\cdots+b_{m} z^{-m} \\
& D\left(z^{-1}\right)=1+d_{1} z^{-1}+d_{2} z^{-2}+\cdots+d_{p} z^{-p} \\
z^{-1} & \text { is the backward shift operator; } y(k-1)=z^{-1} y(k) \\
y & \text { is the process output (measurement) } \\
u & \text { is the process input (control variable) } \\
v & \text { is independent white noise with zero mean } \\
j & \text { is deadtime (number of timesteps lag) } \\
n, m, p & \text { are the polynomial orders }
\end{array}
$$

Introducing a vector $\psi$ containing previous outputs, inputs and noise and a vector $\theta$ containing all parameters, the model (1) is written

$$
y(k)=\psi^{T}(k) \theta(k-1)+v(k)
$$

where

$$
\begin{aligned}
\psi^{T}(k)= & {[-y(k-1) \cdots-y(k-n) u(k-j-1) \cdots} \\
& u(k-j-m) v(k-1) \cdots v(k-p)] \\
\theta^{T}(k-1)= & {\left[a_{1} \cdots a_{n} b_{1} \cdots b_{m} d_{1} \cdots d_{p}\right] }
\end{aligned}
$$

The one-step-ahead prediction error is defined as the difference between the real measurement at time $k+1$ and the prediction at time $k$ of the same measurement.

$$
e(k+1)=y(k+1)-\hat{y}(k+1 \mid k)=y(k+1)-\hat{\psi}^{T}(k+1) \hat{\theta}(k)
$$


Here $\hat{\psi}$ is the estimate of the datavector $\psi$ where the unknown noise signals $v(k-i)$, are substituted by their estimates $e(k-i), i=1, p$. The vector $\hat{\theta}$ contains the parameter estimates.

The new parameter vector is estimated recursively using

$$
\hat{\theta}(k+1)=\hat{\theta}(k)+\gamma(k) e(k+1)
$$

where the gainvector $\gamma$ is given by

$$
\gamma(k)=P(k+1) \hat{\psi}(k+1)=\frac{P(k) \hat{\psi}(k+1)}{\lambda+\hat{\psi}^{T}(k+1) P(k) \hat{\psi}(k+1)}
$$

The matrix $P$ is proportional to the covariance matrix of the parameter estimates and is updated recursively by

$$
P(k+1)=\left[I-\gamma(k) \hat{\psi}^{T}(k+1)\right] P(k) \frac{1}{\lambda}+Q
$$

The $\lambda$ introduced in the above equations is a forgetting factor which makes it possible to track time varying parameters. $\lambda$ equal to 1 means no forgetting, and with $\lambda$ less than 1 the algorithm forgets the past exponentially. Linear forgetting is also possible by adding a constant (diagonal) matrix $Q$ to the $P$ matrix as shown in Eq. (5).

The algorithm described above is valid for the least squares estimation. The recursive maximum likelihood algorithm has the same form, but the data vector $\hat{\psi}$ is filtered through 1/D( $\left.z^{-1}\right)$ before it is used in Eqs. (4) and (5) above, Isermann (1981).

\section{Adaptive control of an alumina reduction cell}

\section{Modelling and parameter estimation}

As mentioned above, it is not yet possible in practice to measure the alumina concentration on-line. However, the concentration has a characteristic influence on the resistance of the cell. This relation is shown qualitatively in Fig. 2.

The relation described in Fig. 2 is not fixed, but will depend on the chemical composition of the bath, on the temperature and, of course, on the interpolar distance. The various curves corresponding to the different combinations of the variables are not known in detail, but they will all have qualitatively the same form with a minimum around $3-4 \%$ alumina. The idea is therefore to estimate the slope of the

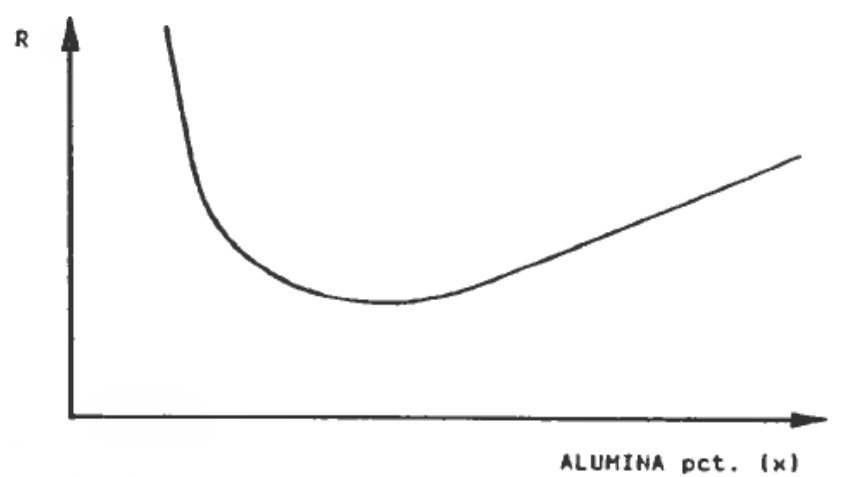

Figure 2. The resistance of the cell versus alumina concentration. 
curve in Fig. 2, based on the resistance measurements, instead of estimating the alumina concentration directly. An estimation of the concentration will require more detailed knowledge about the curves than is available.

If we assume that the resistance is only influenced by the alumina concentration and the anode movement, neglecting the other factors mentioned above, the difference in resistance over the time interval from $k-1$ to $k$ could be written:

$$
R(k)-R(k-1)=b_{1} u_{1}(k-1)+b_{2} u_{2}(k-1)
$$

where

$b_{1}$ is the derivative of the resistance with respect to the alumina concentration

$u_{1}(k-1)$ is the change in alumina concentration in the time interval

$b_{2}$ is the derivative of the resistance with respect to anode movement

$u_{2}(k-1)$ is anode movement in the time interval.

Setting the difference in resistance equal to $y(k)$ and introducing a noise contribution, $n(k)$, we get

$$
y(k)=b_{1} u_{1}(k-1)+b_{2} u_{2}(k-1)+n(k)
$$

$n(k)$ models the noise that affects the system, and we have found it adequate to use simple, first-order representation

$$
n(k)=v(k)+d_{1} v(k-1)
$$

of this noise, where $v(k)$ is an independent white noise sequence. For the RLS estimation method only the simple noise model with $d_{1}=0$ is allowed, while RELS and RML assume the full model in Eq. (7).

If we compare the model (6) with the general model (1), we see that (6) is a two-input, one-output model of first order in $u_{1}, u_{2}$ and $v$ and of zero order in $y$. The algorithm developed for estimation of ARMAX processes could be used unmodified with data vector and parameter vector

$$
\psi(k)=\left[u_{1}(k-1) u_{2}(k-1) v(k-1)\right]^{T}
$$

and

respectively.

$$
\theta(k)=\left[b_{1} b_{2} d_{1}\right]_{k}^{\mathrm{T}}
$$

The input signal $u_{1}(\mathrm{k})$ has to be calculated from the sum of the feedings in the time interval from $k-1$ to $k$ and the estimated alumina consumption in the same interval, i.e.

$$
u_{1}(k)=\text { (feeding rate }- \text { consumption rate) } T / \text { mass-of-bath }
$$

All quantities in the calculation of $u_{1}$ are encumbered by uncertainties. We know the number of feedings to the cell, but not the exact amount of alumina supplied. The consumption rate depends on the current and the current efficiency, the latter being nonmeasurable, but fairly constant. The mass of bath will also vary with time and is not measurable. All these factors make it advantageous to use an estimator to 'measure' the alumina concentration, instead of just keeping account of the alumina supplied and consumed.

The parameter $b_{2}$ depends on the specific resistance in the bath and will therefore vary with the composition and the temperature of the bath. However, the variation of $b_{2}$ is much smaller than of $b_{1}$ and in addition the anode movement $u_{2}$ is 
usually zero, resulting in a somewhat poor estimation of $b_{2}$. It is therefore inadviseable to try to draw too much information from this parameter. To be safe, $b_{2}$ is only allowed to vary between relatively narrow limits.

To make sure that the estimator stays 'awake', a constant diagonal matrix $Q$ is added to the covariance matrix $P$ as in Eq. (5). The advantage of this compared to using a forgetting factor $\lambda$, is that different forgetting may be put on the parameters. If the feeding of the cell is such that the variation in alumina concentration is large, one will expect considerable variations in $b_{1}$. The element in $Q$ that corresponds to $b_{1}$ has to be relatively large for the estimator to be able to follow these variations. The disadvantage of using a too fast forgetting is that the estimator becomes sensitive to noise and will give a poorer filtering of the data.

To make the estimator capable of handling sudden spikes in the resistance, a filtering of the estimation error is included. This filtering is done by modifying the gain vector in Eq. (4) as follows:

$$
\gamma(k)_{\bmod }=\gamma(k) / f(k)
$$

where

$$
\begin{aligned}
& f(k)=\max \left(|e(k)| / e_{f}(k), 1 \cdot 0\right) \\
& e_{f}(k)=\alpha e_{f}(k-1)+(1-\alpha) \beta|e(k-1)| \\
& e(k) \quad \text { is estimation error } \\
& \alpha \quad \text { is a filtering factor, } \alpha<1 \cdot 0 \\
& \beta \quad \text { is a constant, preferably } \approx 2 \cdot 0
\end{aligned}
$$

This filtering will effectively damp out estimation errors which are much larger than a weighted average of $\beta$ times the latest errors.

Some cells exhibit more high frequency noise in the resistance than others. For these cells a prefiltering of the resistance through a low-pass filter may be advantageous. This possibility is included in the input part of the estimator.

\section{The controller}

The controller can manipulate the two control variables $u_{1}$ and $u_{2}$ by ordering different feedings of alumina and movements of the anode respectively. The fact that the alumina concentration may vary over the interval 2 to $5 \%$, allows for relatively large variations in the feeding, and therefore a 'persistently exciting' control signal $u_{1}$ may be generated to ensure reliable $b_{1}$ estimates.

The control problem is solved in the following way. In the normal mode the output to the alumina feeder has two levels; one overfeeds the cell by a certain percentage, and the other underfeeds the cell by the same (or another) percentage. However, the actual amount of alumina representing the two levels is not fixed, but is adjusted in accordance with the mass balance of the cell. The shifting from overfeeding to underfeeding and vice versa is based on the estimated value of the $b_{1}$ parameter. When, during underfeeding, the value of $b_{1}$ becomes lower than $b_{10}$, overfeeding takes over and vice versa. To avoid a too frequent shifting caused, for example, by noise in the calculation of $b_{1}$, a dead band is used for the first samples after a shift, as illustrated in Fig. 3. The dead band is wide immediately after the shift and decreases to zero after some samples.

The mean operating point of alumina concentration is the point on the curve in Fig. 2 that corresponds to the slope $b_{10}$. Normally $b_{10}$ is given a small negative 


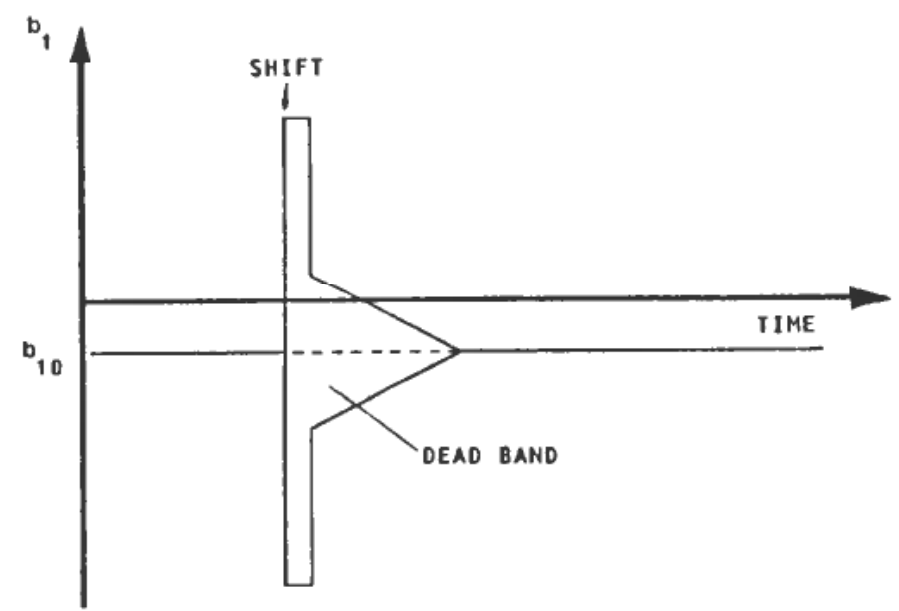

Figure 3. Limits for shifting from over- to underfeeding and vv.

value. The advantage of this choice is that the alumina concentration will vary in an interval of the curve where the response in the resistance is best, and also the mean concentration will be relatively low and thus reduce the risk of sludge deposition. During the test period reported below, the operation point was $b_{1}=0$, but later a small negative value has been used.

The control of the anode position $u_{2}$ is also attached to the $b_{1}$ parameter. The resistance is allowed to vary within a band on each side of the reference value without any anode control. The width of this band depends on the value of $b_{1}$ as shown qualitatively in Fig. 4.

During normal, stable operation of the cell, only a small number of anode movements per day are necessary to satisfy the above criterion. This implies that the estimator is very weakly excitated by the control signal $u_{2}$, and the estimation of $b_{2}$ is therefore poor. Since $u_{2}$ is usually zero, this will only have minor influence on the estimation of $b_{1}$.

\section{Supervisory level}

An alumina reduction cell is subject to severe disturbances even under normal operation. These are caused by tapping of aluminium, change of anode carbons,

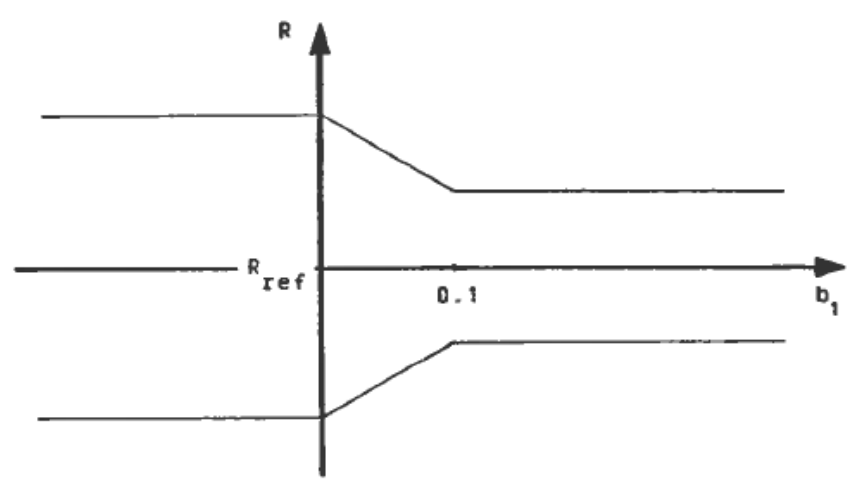

Figure 4. Resistance variation band. Anode control outside band. 
anode rack raising, current disconnection and anode effect. These disturbances result in variations in the resistance which could be erroneously interpreted by the estimator. To overcome this problem, a supervisory level is included in the control system. This supervisor stops the estimator during such disturbances and provides for restart with reasonable initial values a suitable time interval after the disturbance is over. In addition, it will order different alumina feedings during this period dependent on the type of disturbance. The various disturbances mentioned above, are identified either manually by push-buttons or automatically by testing of the voltage and current measurements.

To dissolve sludge which may deposit during overfeeding or from some of the above disturbances and to guarantee that the alumina concentration is kept under control even if the estimator fails, the supervisor initiates a tracking mode at certain time intervals. During tracking the feeding of alumina is very low, and the course of the resistance is tracked towards the anode effect. When the slope of the resistance curve, calculated by linear regression, exceeds a prespecified value, the cell is assumed to be close to the anode effect, and the feeding of alumina is restarted. Normally, the cell is supplied with an extra amount of alumina during the first minutes after this restart to avoid the anode effect.

The amount of alumina in each addition is recalculated after each tracking period. This is done by counting the number of additions supplied since the last tracking and calculating the amount of alumina consumed. Assuming that the alumina concentration is the same at the end of each tracking period, it is easy to calculate the average alumina addition. By these recalculations it is possible to correct the additions of alumina according to changes in the quality of the alumina. This is necessary when a volumetric feeder is used.

\section{Case studies}

We will now describe the results of some estimations done offline, but on data taken from a period where the controller was run on one cell. The resistance and the two control signals were recorded, and the alumina concentration was measured manually. The duration of this test period was 320 samples, and with a sampling time of 5.5 minutes this means about 29 hours. The sampling time was found to be short enough to identify the interesting dynamics of the process.

The resistance and the two control signals are shown in the upper part of Fig. 5. The test period included two events; a tapping of aluminium at $k \approx 80$ and a change of anode carbon at $k \approx 240$. The disturbance to the resistance during these events is easily seen. In addition, tracking was initiated at $k=133$ and lasted until $k=143$. The ripple in the $u_{1}$ signal is due to the discrete nature of the feeding, which causes small differences in the number of alumina additions in the various timesteps, even if the feeding frequency is constant.

Parameter estimation using the methods RLS, RELS and RML has been compared with respect to the variance of the estimation error. The RML method was found to be slightly better than the RELS method, which was somewhat better than the RLS. As a consequence of this investigation, the RML method is presently used in the on-line system.

In the lower part of Fig. 5 are shown the results of the parameter estimation using the RML method, together with the measured alumina concentration. With reference to the resistance vs. alumina concentration curve, Fig. 2, we would expect 


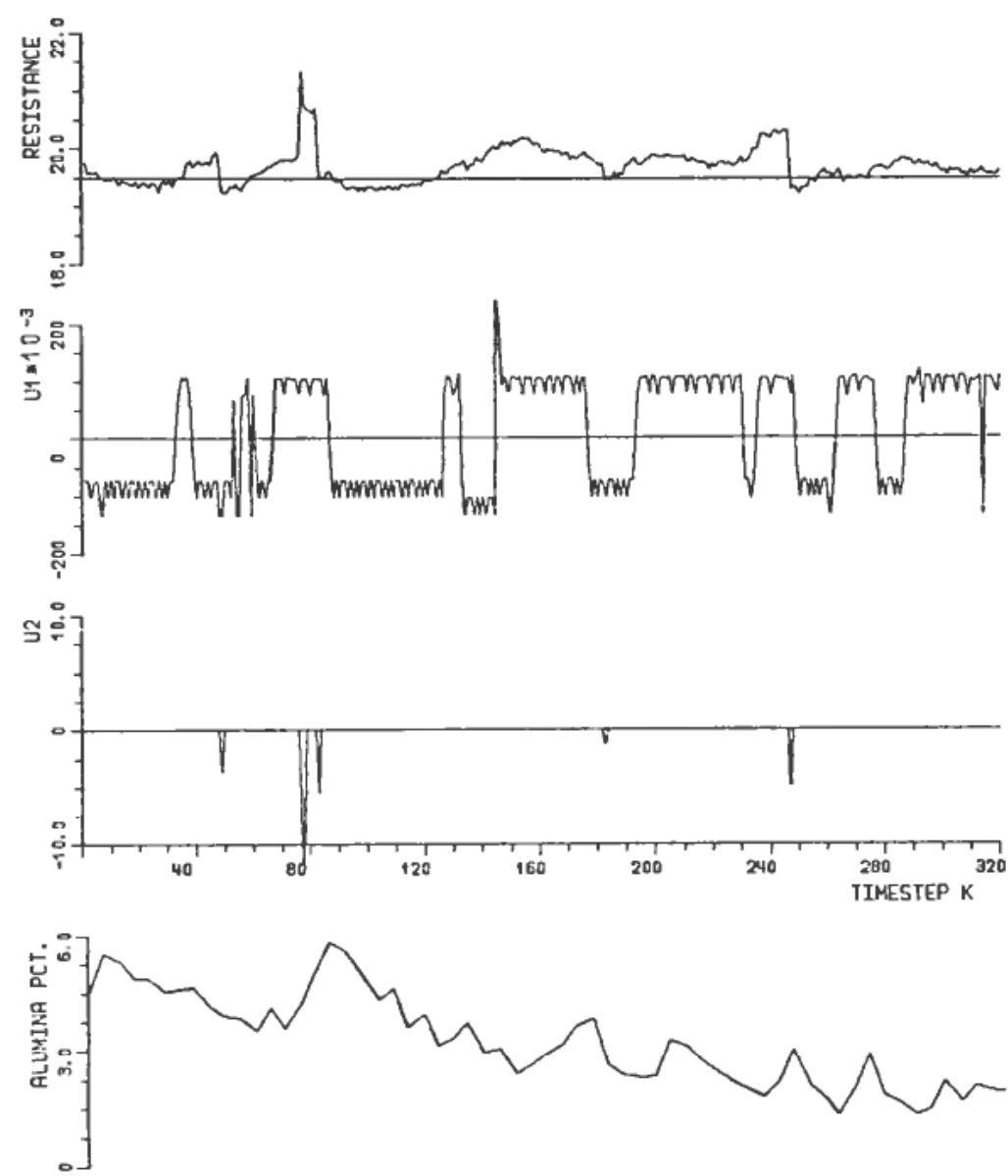

(a)

(b)

(c)

(d)

$\frac{?}{0}$

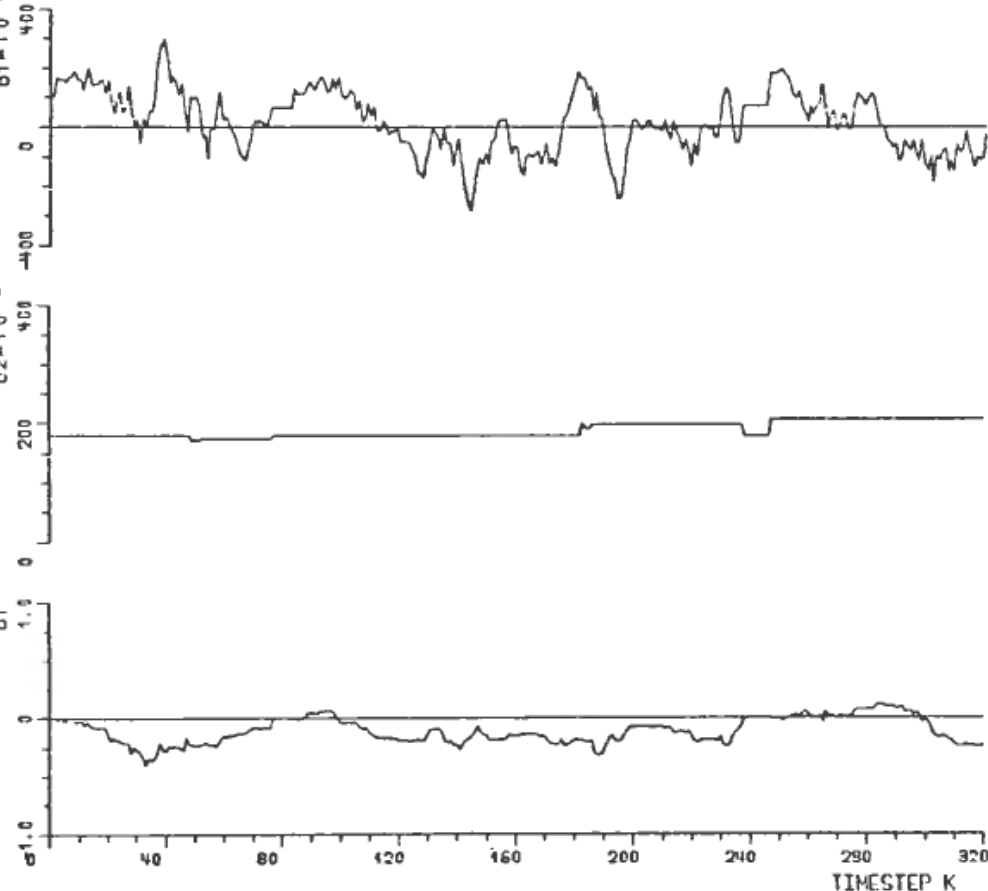

(e)

(g)

Figure 5. Results of estimation using the RML method. 
the $b_{1}$ estimates and the alumina concentration to be positively correlated. This is true for the first half of the period, but the decreasing trend in the alumina concentration does not show up in the $b_{1}$ estimates.

The reason for this trend is that the feeder mechanism failed during the last part of the test period, and the cell was supplied with much less alumina than $u_{1}$ says. This resulted in an apparent overfeeding in the last part of the period, as Fig. 5 shows.

The estimation algorithm requires that the mean value of $u_{1}$ is zero. This is normally satisfied, at least approximately, in our system. When the feeding mechanism fails, however, the mean value of $u_{1}$ will differ from zero and show an increasing trend. This will result in biased estimates as is seen in Fig. 5e. In spite of this fact, the controller works fairly well during this period by ordering overfeeding of the cell most of the time.

We may overcome the above problem to some extent by including a mean value compensation in the algorithm. The mean value of $u_{1}$ is then calculated with an exponential forgetting.

$$
\bar{u}_{1}(k)=\lambda \bar{u}_{1}(k-1)+(1-\lambda) u_{1}(k)
$$

where $\lambda$ is the forgetting factor, less than, but close to 1 . The control signal is modified to

$$
u_{1}(k)^{\prime}=u_{1}(k)-\bar{u}_{1}(k)
$$

The modified control signal $u_{1}(k)^{\prime}$ is then input to the estimator. The results of the estimation using this modified algorithm, are shown in Fig. 6. We see here the
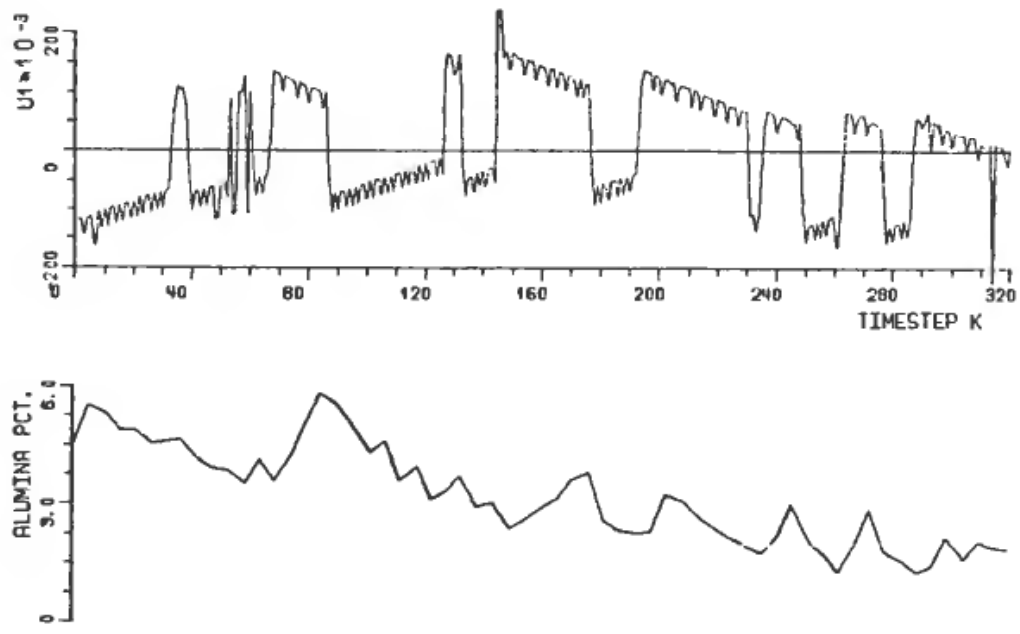

(b)

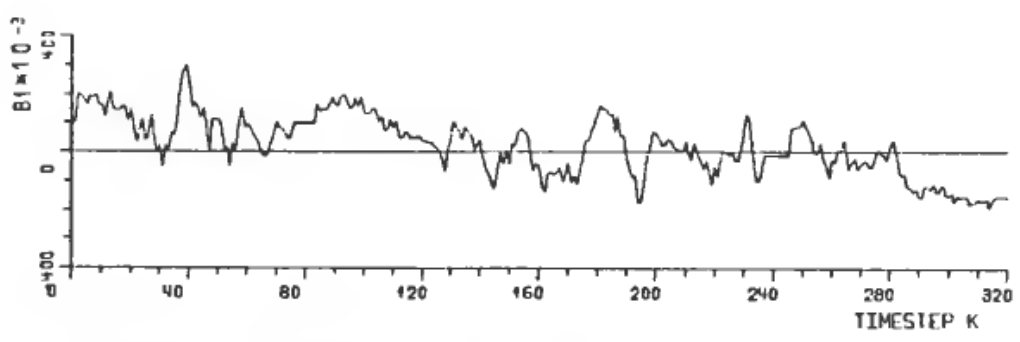

(c)

Figure 6. Estimation with mean-value compensation in control signal. 
same decreasing trend in the $b_{1}$ estimate as in the alumina concentration, due to the decreasing level of $u_{1}^{\prime}$. This modification of the algorithm may be useful to improve the estimator's behaviour during periods with failures in the feeder system, but has not yet been tested on-line.

\section{Experiences and results from the long-term testing}

When the test cells were converted to point feeder technology, equipment was installed for alumina feeding at two points. In addition, equipment was installed for automatic anode effect quenching, but the heat balance and the cathode lining of these cells were unchanged.

The test cells have been operated on $153 \mathrm{kA}$. They are arranged end-to-end and contain 20 anode carbons, with an anodic current density of $0.8 \mathrm{~A} / \mathrm{cm}^{2}$. The anodes have been covered with alumina and the butts have been cleaned inside the cells.

The control system was implemented on local microcomputers at each cell. In addition to the controller algorithm described here, the microcomputer handles all on-line measuring and output to the cell. Communication between the operator and the microcomputer is done via a panel and a display at the front of the control cabinet. The microcomputers also communicate with a central computer which handles long term storing of data.

The controller has been run on the test cells for a period of nearly 3 years, and important data has been recorded frequently.

During the first part of this test period there was an improvement in current efficiency, but nearly no effect on the energy consumption. Some practical problems associated with the operation of the cell were clarified, like the $\mathrm{AlF}_{3}$ additions to point feeder cells. This experience lead to a rebuilding of the test cells. Equipment was installed for automatic $\mathrm{AlF}_{3}$ additions similar to the alumina feeders, and the heat balance of the cells was changed by relining of the cathode. This relining was done on the basis of calculations made by ÅSV's mathematical models for heat balance.

The results from the test period are divided into two parts. The first part shows the results after the first rebuilding, and the second part the results after the second rebuilding. The average bath composition over the whole test period has been 10 wt $\%$ excess $\mathrm{AlF}_{3}$ and $5 \mathrm{wt} \% \mathrm{CaF}_{2}$.

Part 1, period of nearly 2 years:

Average bath temperature $\ldots \ldots \ldots \ldots \ldots \ldots \ldots \ldots \ldots \ldots, 961^{\circ} \mathrm{C}$

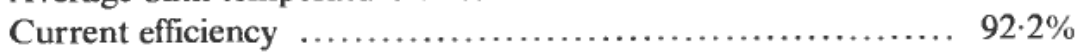

Energy consumption ............................. $15 \cdot 5 \mathrm{kWh} / \mathrm{kg} \mathrm{Al}$

Part 2, period of about 9 months:

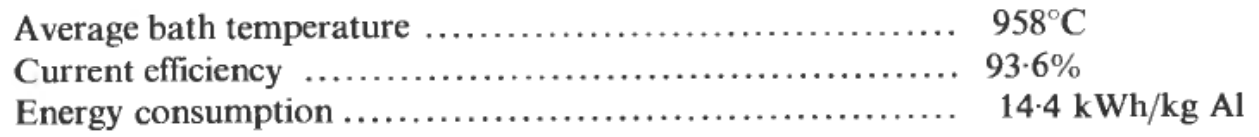

The controller has reduced the anode effect frequency to only 0.12 per day or about once every 8th day. The average alumina concentration in the bath was measured to $3.1 \pm 0.7 \mathrm{wt} \%$. (Operating point $b_{10}=0$ ). 
During the test period there have been frequent changes in the alumina qualities, but these variations have been handled in a satisfactory way by the controller, and have apparently not influenced the cell in a negative way.

It is difficult to judge how much of the above improvements stem from the rebuilding of the cell and how much is due to the adaptive controller itself. However, the adaptive feature of the controller makes it possible to operate the cell within a favourable alumina concentration range, which otherwise would have been difficult due to the lack of information about this concentration. This stabilizes the cell, leading to a higher current efficiency. It is therefore reasonable to assert that most of the improvements stated above are due to the controller design in combination with the point feeder technique.

\section{Conclusion}

The paper describes a control system for alumina reduction cells using point feeder technology. The system controls the alumina concentration and the energy input (resistance) of the cell. The system has been tested for almost 3 years and is today in operation on several cells in A/S Årdal og Sunndal Verk's aluminium works. The results so far seem promising, although the work is just a first step towards the final goal; a full mass- and energy balance control system for the alumina reduction cells.

\section{REFERENCES}

GRAN, E. (1980). Multivariable control in aluminium reduction cells. Modelling, Identification and Control, 247-258.

Isermann, R. (1981). Digital Control Systems (Springer Verlag: Berlin).

LjUnG, L. and SøDerstrøm, T. (1983). Theory and Practice of Recursive Identification (The MIT Press: Cambridge, Mass.). 